\title{
ARE TWO VIEWS OF THE PELVIS ROUTINELY REQUIRED IN THE ASSESSMENT OF THE LIMPING CHILD?
}

\author{
Abeynayake S D'ㄹ, Jackson $\mathrm{M} \mathrm{R}^{2}$ \\ ${ }^{1}$ Colombo North Teaching Hospital, Ragama, Sri Lanka \\ ${ }^{2}$ Royal Victoria Infirmary, Newcastle Upon Tyne, United Kingdom
}

\begin{abstract}
Introduction: Atraumatic hip pain presenting with a limp is a common clinical problem in children. It is considered a diagnostic challenge to the physician.

Objectives: Aim of this study was to assess the sensitivity of a single view of the pelvis in radiographic imaging of a limping child.

Methodology: Retrospective analysis of all paediatric pelvic radiographs performed in Great North Children's Hospital, Royal Victoria Infirmary, Newcastle upon Tyne, UK from 2004 to 2014 was carried out. 143 cases were recruited, in whom both AP and FL views had been performed at presentation, and a cause for hip pain had been identified based on the radiological report. These 286 radiographs were anonymised and randomly shuffled with 100 age-matched controls (50 AP / 50 frog-lateral) selected from normal radiographs within the same period to avoid bias. The images were independently reviewed by three consultant paediatric radiologists.

Results: Perthes/Avascular necrosis (AVN) (n=117) was identified with $94.6 \%$ sensitivity on the AP view compared to $94.3 \%$ on the frog-lateral view. Sensitivity for Slipped Upper Femoral Epiphysis (SUFE) $(n=19)$ was $75.4 \%$ on AP and $100 \%$ on frog-lateral. Seven cases of other pathologies were identified with sensitivity of $61.9 \%$ on AP and $57.2 \%$ on frog-lateral.

Conclusion: Frog lateral view is superior to AP view in detection of SUFE and comparable in sensitivity for Perthes to the AP view. We suggest only the frog-lateral view is required in the routine assessment of the limping child.
\end{abstract}

Key words: Pelvic Radiographs, Limping child, Atraumatic hip pain

Corresponding author: Abeynayake S D, e mail: snjabey@hotmail.com

Copyright: Abeynayake S D 


\section{Background}

Acute atraumatic hip pain is a common presentation in children and a child with a limp is a diagnostic challenge to the physician. Children with hip pathology can present in different ways, including pain, refusal to bear weight, limp or abnormal gait or decreased movement of the lower extremity ${ }^{1}$. The differential diagnosis is extensive but the aetiology may vary according to the age of the child ${ }^{2}$. In most cases the diagnosis can be made by history and proper physical examination.

Ultrasound should be the first line imaging investigation in a child with an atraumatic $\operatorname{limp}^{3}$. However, while ultrasound can offer excellent visualisation of a hip joint effusion, it offers limited characterisation of the bones. Conventional plain radiography is therefore commonly used in the investigation of painful hip or limp, either in addition to or without the use of ultrasound according to local protocols. MRI can be considered the gold standard imaging modality for the investigation of paediatric hip pathology, offering excellent characterisation of both bone and soft tissue without utilisingionising radiation. MRI may show proximal femoral or pelvic pathology not visible on plain radiograph including early evidence of avascular necrosis, osteomyelitis or rarer cause of hip pain including bone tumours ${ }^{4}$. However, MRI is not freely available in most institutions and may require general anaesthesia in younger patients.
The causes of acute non-traumatic childhood hip pathology vary from quite harmless conditions such as transient synovitis of the hip to more severe problems such as Perthes disease, slipped upper femoral epiphysis (SUFE) and potentially life-threatening conditions like septic arthritis or osteomyelitis $^{5}$. Perthes disease and SUFE are the commonly encountered conditions in a limping child.

Perthes disease refers to idiopathic avascular necrosis (AVN) of the femoral epiphysis seen in children. Boys are more likely to be affected than girls. Presentation is typically at a younger age than SUFE with peak presentation at 5-6 years, but confidence intervals are as wide as 2-14 years ${ }^{4}$. The earliest radiographic feature is radiolucent subchondral fissure, described as the crescent sign. With disease progression there will be loss of height, fragmentation and sclerosis of the femoral head epiphysis ${ }^{6}$.

SUFE is the most common hip abnormality in adolescents ${ }^{7}$. It is more common in boys and the age range is 11-14 years at the time of pubertal growth spurt. In radiography it is diagnosed with Klein's line drawn in the Anterior-Posterior (AP) view and with the slip angle measured on the frog-lateral (FL) view $^{6}$. In early SUFE, AP radiograph may be normal as the slip is initially in a posterior direction.

The effects of medical radiation exposure in childhood can last a lifetime and therefore concerns have been raised about the potential harm from early medical 
irradiation $^{8}$.

Many centres routinely perform both AP and FL views in the assessment of the limping child as it has been considered more reliable in achieving the diagnosis irrespective of the radiation hazard ${ }^{9}$. However there is recent evidence to suggest one view of the pelvis is sufficient for assessing children presenting with hip pain $^{10}$, and several paediatriccentresin the UK currently advocate this approach.

The aim of this study was to assess the sensitivity of a single view in radiographic imaging of the pelvis to diagnose hip related disease conditions in children.

\section{Methods and Materials}

A retrospective analysis of all paediatric pelvic radiographs $(n=6135)$, performed following hip pain in children and available in the archives of Great North Children's Hospital, Royal Victoria Infirmary, Newcastle Upon Tyne, United Kingdom during the period of 2004 to 2014, was carried out. Exclusion criteria were those examinations where only one view, either AP or FL view had been performed; if they were follow up images; images of development dysplasia of hip (DDH) or fractures and images of extremely poor quality.

Accordingly, a total of 143 digital radiographic examinations of children were identified in whom both AP and frog-lateral views had been performed at presentation and a cause for hip pain had been identified based on the radiological report. These 286 radiographs were then anonymised and randomly shuffled with another 100 agematched control radiographs of children which were normal pelvic radiographs (50 $\mathrm{AP}$ and $50 \mathrm{FL}$ ), performed during the same period in the same institution. All these radiographs were arranged into a power point presentation and were independently reviewed by three consultant paediatric radiologists to make a diagnosis related to the hip pain and their results were analysed.

\section{Data analysis}

The sensitivity of only one radiographic view (either AP or FL) in making a diagnosis of hip related disease condition was assessed against the gold standard, which was the diagnosis made using both $\mathrm{AP}$ and FL views.

\section{Results}

Out of these 143 plain radiographic examinations carried out in children, there were 117 with Perthes / AVN, 19 with SUFE and 7 cases of other bone pathology. Eighty seven(75\%) were males and 30 (25\%) were females in the Perthes /AVN group and 11 (58\%) were males and $8(42 \%)$ were females in the SUFE cases. Majority (77\%) of the individuals with AVN were between the ages of 3 and 9 years, while all the individuals presented with SUFE were between 9 and 14 years of age (Table 1 and Table 2). 
Table 1 - Age distribution at presentation of Perthes / AVN

\begin{tabular}{|l|l|l|l|l|l|l|l|l|l|l|l|l|}
\hline $\begin{array}{l}\text { Age } \\
\text { (years) }\end{array}$ & $2-3$ & $3-4$ & $4-5$ & $5-6$ & $6-7$ & $7-8$ & $8-9$ & $9-10$ & $10-11$ & $11-12$ & $12-13$ & $13-14$ \\
\hline Number & 1 & 17 & 16 & 12 & 13 & 11 & 21 & 4 & 9 & 7 & 2 & 4 \\
\hline
\end{tabular}

Table 2 - Age distribution at presentation of SUFE

\begin{tabular}{|l|l|l|l|l|l|}
\hline $\begin{array}{l}\text { Age } \\
\text { (years) }\end{array}$ & $9-10$ & $10-11$ & $11-12$ & $12-13$ & $13-14$ \\
\hline Number & 2 & 3 & 5 & 6 & 3 \\
\hline
\end{tabular}

Table 3-Degree of agreement of interpreters on pathological conditions considered

\begin{tabular}{|l|l|l|l|ll|ll|}
\hline Disease condition & View & \multicolumn{2}{|l|}{$\begin{array}{l}\text { EXAMINER } \\
\text { A }\end{array}$} & \multicolumn{2}{|l|}{$\begin{array}{l}\text { EXAMINER } \\
\text { B }\end{array}$} & \multicolumn{2}{|l|}{ EXAMINER } \\
\hline Perthes / AVN (n=117) & AP & \multicolumn{1}{|l|}{109} & $(93 \%)$ & 110 & $(94 \%)$ & 113 & $(97 \%)$ \\
\hline & FL & 108 & $(92 \%)$ & 111 & $(95 \%)$ & 112 & $(96 \%)$ \\
\hline SUFE (n=19) & AP & 16 & $(84 \%)$ & 13 & $(68 \%)$ & 14 & $(73 \%)$ \\
\hline & FL & 19 & $(100 \%)$ & 19 & $(100 \%)$ & 19 & $(100 \%)$ \\
\hline Other Pathology (n=7) & AP & 4 & $(57 \%)$ & 5 & $(71 \%)$ & 4 & $(57 \%)$ \\
\hline & FL & 4 & $(57 \%)$ & 4 & $(57 \%)$ & 4 & $(57 \%)$ \\
\hline Normal (n=50) & AP & 50 & $(100 \%)$ & 50 & $(100 \%)$ & 46 & $(92 \%)$ \\
\hline & FL & 50 & $(100 \%)$ & 50 & $(100 \%)$ & 47 & $(94 \%)$ \\
\hline
\end{tabular}

Sensitivity of making the diagnosis of SUFE was $75.4 \%$ on AP view alone and $100 \%$ on FL view alone. Sensitivity of making a diagnosis of Perthes/AVN was $94.6 \%$ on the AP pelvic view alone compared to $94.3 \%$ on the FL view alone. However sensitivity with both views when combined was $97.7 \%$ (Table 3). Sensitivity of making a diagnosis of other pathologies was $61.9 \%$ on AP view alone and $57.2 \%$ on the FL view alone.

\section{Limitations of the Study}

Radiographic reporting conditions of the study for the examiners did not replicate normal reporting environment. AP and FL views were separated, images were presented as power point slides with no facility to zoom or change the windowing, 
and a large number of views had to be reviewed in a very short period.

The accuracy of the original report was assumed to be correct and considered as the gold standard in this study.

Potential scorer bias cannot be excluded as one examiner was involved in the study design and could have been motivated to miss pathology on one type of view to help support the hypothesis. However, it is hoped that this potential bias was minimal with the scorers motivation to perform well in competition with each other, and that the use of three independent scorers was helpful in reducing this observation bias.

Specificity and other validity checks were not carried out as the diagnosis of the said pathologies are made solely on plain radiography in the current practice of imaging even though advanced imaging such as MRI are available.

\section{Discussion}

Perthes disease and SUFE are common childhood diseases, which typically present with hip pain or limping. Both these conditions are commoner in males and similar finding was found in this study as well. Majority of children with both Perthes/AVN (3:1) and SUFE (3:2) were males, which was compatible with the general population ${ }^{6}$. The majority of children presented with AVN were between the ages of 3 and 9 years and all the children presented with SUFE were between 9 and
14 years in this study, which is also compatible with usual ages of presentation shown in other studies ${ }^{4,7}$.

The first line imaging modality in the limping child is ultrasound ${ }^{11}$. It will help to confirm a joint effusion and help to diagnose transient synovitis or septic arthritis. If the ultrasound is normal and/or symptoms persist, then conventional radiograph of the pelvis may help demonstrate a vascular necrosis, osteomyelitis or other diseases such as tumours. It is important to recognise that MRI offers superior sensitivity and specificity for all of these ${ }^{12,13}$, with the advantage of no ionising radiation. Centres which are able to offer ready access to MRI may be able to "bypass" the use of plain radiographs altogether in some cases. However, we have observed that many centres are not in this position, and routinely perform both AP and FL.

Nnadi et al. compared the sensitivity and specificity of a single AP and a single FL view with the combination of $\mathrm{AP}$ and FL views in a group of 96 children, and found the combination to be superior to either single view ${ }^{14}$. Although combination of AP and FL has been perceived to offer high diagnostic accuracy, it effectively doubles the radiation exposure ${ }^{9}$ even though gonad shielding may partly reduce the dose of the second view ${ }^{15}$. However gonadal shield placement is not universally practised in a limping child as it may obscure detail and it is considered controversial ${ }^{16}$. The effects of medical radiation exposure in childhood can 
last a lifetime and it is the primary responsibility of the diagnostic imaging personnel to take measures to reduce radiation of children. Effective dose for a AP pelvis single radiograph is $0.7 \mathrm{mSv}$ which is 35 times higher compared to a PA Chest $\mathrm{X}$ ray $(0.02 \mathrm{mSv})^{9}$. Tissues of children are more sensitive to ionising radiation and risk of radiation induced carcinogenesis is greater in them as children have more rapidly dividing cells and they have longer expected lifetime for the effects of radiation exposure to manifest as cancer. According to ALARA principle unnecessary radiation exposure during medical procedures should be avoided. This should be particularlyobserved when the patient is a child $^{17}$.

This study demonstrates that the frog-leg lateral view is superior to AP in the detection of SUFE and comparable in sensitivity for Perthes /AVN to the AP view. Performing a solitary FL radiograph would substantially reduce the radiation exposure as well as the cost of imaging. Some radiologists have suggested modification of Klein's line to improve the sensitivity of AP view in the diagnosis of $\mathrm{SUFE}^{18}$ but this cannot be replaced without FL view for accurate diagnosis.

In our study, performing both views did offer a modest benefit over a single view as shown by sensitivity for AVN (97.7\% for both views compared to $94.3 \%$ for frog leg view alone) but effectively doubles the radiation dose. Bomeret al. compared the sensitivity and specificity of single FL radiograph with the combination of $\mathrm{AP}$ and FL radiographs in a group of 524 children and proved that solitary FL radiograph is as accurate as the combined views in diagnosing hip disease in children. $\mathrm{He}$ further emphasized that the majority $(91 \%)$ of the radiographs performed in hip pain were normal ${ }^{10}$. Therefore performing both views would needlessly irradiate large number of normal children for a very modest increase in sensitivity.

Some radiologists suggest that the AP view is better than FL view for AVN, as they feel more comfortable in reporting with AP view in day to day practice, but this study did not support this evidence. Our study showed both views are comparable in sensitivity in the diagnosis of AVN.

Other lesions which were evident on radiographs in the study were chondroma of inferior pubic ramus, osteomyelitis of left femoral head, subchondral cyst of acetabulum, osteolytic lesion of greater trochanter, osteopetrosis, osteoid osteoma of L/femoral diaphysis and aneurysmal bone cyst of femoral neck. These were subtle lesions and some were not diagnosed accurately by the interpreters. However their original diagnosis was with the MRI in addition to plain radiography. MRI is the gold standard for infection and bone tumours $^{13}$, therefore plain radiography should not be performed alone, when there is a suspicion of a tumour or an infection. 
In centres with limited access to MRI, there may be a role for the AP view in younger children, but it should not be performed routinely in children over 9 years of age. Either AP or FL view could be considered according to their preference in younger children. If both views are felt to be required, a delay should be considered between each radiograph to allow an opportunity for self-limiting causes of hip pain, such as transient synovitis to resolve. Provided appropriate follow up is arranged, it is unlikely that a child presenting early with AVN will come to harm by being managed as though they have transient synovitis for a few days.

\section{Conclusion}

Frog lateral view is found to be superior to AP view in diagnosis of slipped upper femoral epiphysis and comparable in sensitivity for avascular necrosis to the AP view in this study. Therefore we suggest that only the frog-lateral view is required in the routine assessment of the limping child.

\section{Acknowledgements}

We thank Claire Gowdy and Deniz Morgan for their contribution on image interpretation.

\section{References}

1. Frick SL Evaluation of the child who has hip pain. Orthopaedic Clinicsof North America 2006; 37:133-140.

2. Leung AK, Lemay JF. The limping child.Journal of Pediatric Health Care. 2004; 18(5):219-23.

3. LeQuesne . A role for ultrasound in assessment of the child with a painful limp.Journal of Paediatric Child Health.1993; 29(4):247-9.

4. Dillman JR, Hernandez RJ. MRI of Legg-Calve-Perthes disease. American Journal of Roentgenology. 2009; 193(5):1394-407.

5. Krul M, Wouden JC, Schellevis FG, Suijlekom-Smit LWA, KoesBW . Acute non-traumatic hip pathology in children: incidence and presentation in family practice. Family Practioner 2010; 27:166-170.

6. Adam A., Dixon A. K., Grainger R. G., \& Allison D. J. Grainger \& Allison's diagnostic radiology: A textbook of medical imaging 2015; 79: 1916-18.

7. Boles CA, El-khoury GY. Slipped capital femoral epiphysis. Radiographics.; 17 (4): 809-23.

8. Robinow M, Silverman FN. Radiation hazards in the field of Paediatrics. Paediatrics.1957; 20:921-940. 
9. Wall BF, Hart D. Revised radiation doses for typical x-ray examinations. The British Journal of Radiology 1997; 70:437-439.

10. Bomer J, Klerx-Melis F, Holscher HC (2014) Painful paediatric hip: frog-leg lateral view only. European Radiolology. 2014; 24:703-708.

11. Petkovic L, Maric D, GajdobranskiD .Ultrasonographic differentiation of painful hip in developmental age. Medical Pregl. 2010; 63(3-4):208-14.

12. Coleman BG, Kressel HY, Dalinka MK, Scheibler ML, Burk DL, Cohen EK. Radiographically negative avascular necrosis: detection with MR imaging.Radiology.1988; 168(2):525-8.

13. Glickstein MF, Burk DL Jr, Schiebler ML, Cohen EK, Dalinka MK, Steinberg ME, Kressel HY. Avascular necrosis versus other diseases of the hip: sensitivity of MR imaging.Radiolog 1988; 169(1):213-5.

14. Nnadi C, Chawla T, Redfern A, Argent J, Fairhurst J, Clarke N . Radiograph evaluation in children with acute hip pain. Journal of PediatricOrthopaedics 2002; 22:342-344.

15. Sikand M, Stinchcombe S, Livesley P J. Study on the use of gonadal protection shields during paediatric pelvic X-rays. Annals of Royal College of Surgeons England.2003;85(6):422-5.

16. Frantzen MJ, Robben S, Postma AA, Zoetelief J, Wildberger JE, Kemerink GJ . Gonad shielding in paediatric pelvic radiography: disadvantages prevail over benefit. Insights Imaging 2012; 3:23-32.

17. Furlow B. Radiation protection in pediatric imaging. Radiolology Technologist. 2011; 82:421-439.

18. Green DW, Mogekwu N, Scher DM, Handler S, Chalmers P, WidmannRF .A modification of Klein's Line to improve sensitivity of the anterior-posterior radiograph in slipped capital femoral epiphysis. J Pediatric Orthopaedics. 2009; 29(5): 449-53. 